\title{
EVALUATION DU DEVELOPPEMENT COGNITIF ET DES FACTEURS PSYCHOSOCIAUX DE MAUVAISE ADAPTATION CHEZ LES SCHIZOPHRENES TRAITES AU CENTRE NEURO-PSYCHO-PATHOLOGIQUE «C.N.P.P. » DE L'UNIVERSITE DE KINSHASA, DEMOCRATIC REPUBLIC OF THE CONGO
}

\author{
Tshibwabwa Popi René1i, \\ Mukau Ebwel Joacim², \\ Yawidi Mayinzambi Jean-Paul', \\ Okitundu Daniel $^{4}$ \\ ${ }^{1}$ Chef de Travaux à la Faculté de Psychologie \\ et des Sciences de l'Education, \\ Université Pédagogique Nationale, \\ Kinshasa, République Démocratique du Congo (RDC) \\ 2Professeur, \\ Université Pédagogique National, \\ Kinshasa, République, Démocratique du Congo (RDC) \\ ${ }^{3}$ Professeur, \\ Université Pédagogique Nationale, \\ Kinshasa, République Démocratique du Congo (RDC) \\ ${ }^{4}$ Professeur, \\ Université de Kinshasa, \\ Kinshasa, République Démocratique du Congo (RDC)
}

\begin{abstract}
:
Cette étude a évalué les déficits des fonctions neurocognitives de base, les facteurs psychologiques et ceux de la sphère sociale associés qui interfèrent avec la mauvaise adaptation des patients schizophrènes traités au C.N.P.P. de l’Université Kinshasa. Elle a fait suite à une recherche initiale menée auprès de 48 personnes souffrant de schizophrénie et qui avaient montré après la stabilisation des symptômes cliniques, la difficulté d'interagir adéquatement avec la réalité était la plainte. Le suivi psychologique en postcure a révélé une mauvaise intégration de ces patients dans le contexte familial. Quatre patients issus de cette cohorte, sélectionnés par choix raisonné ou de convenance (Pirès, 1977) ont été évalués à l'aide d'entretien clinique, des tests neuropsychologiques et des échelles de mesure sociale. L'objectif a consisté à utiliser une approche de remédiation cognitive de restauration du processus adaptée aux difficultés et des interventions individualisées de réadaptation psychosociale pouvant favoriser
\end{abstract}

${ }^{i}$ Correspondence: email tshibwabwa.rene@gmail.com 
Tshibwabwa Popi René, Mukau Ebwel Joacim, Yawidi Mayinzambi Jean-Paul, Okitundu Daniel

EVALUATION DU DEVELOPPEMENT COGNITIF ET DES FACTEURS PSYCHOSOCIAUX

DE MAUVAISE ADAPTATION CHEZ LES SCHIZOPHRENES TRAITES AU CENTRE NEURO-PSYCHO-

PATHOLOGIQUE «C.N.P.P. » DE L'UNIVERSITE DE KINSHASA, DEMOCRATIC REPUBLIC OF THE CONGO

l'intégration de la personne à la famille et à la communauté, au rythme individuel. Les résultats des bilans individuels réalisés ont révélé des profils neurocognitifs déficitaires de mémoires, d'attention et de planification des tâches, un coping inapproprié, une estime de soi faible, une accumulation des stress, la stratégie globale de résolution de problèmes, un soutien familial non perçu, une relation malade-médecin moins bonne, une attribution causale externe de la maladie, une habitude de consommation de substances psychoactives, l'appartenance de l'enfant à une famille désunie.

Keywords: schizophrénie, déficits neurocognitifs, facteurs psychosociaux de mauvaise adaptation, approche de remédiation cognitive, interventions de réadaptation individualisée

\section{Introduction}

Les observations cliniques réalisées auprès de 48 patients souffrant de schizophrénie pris en charge au Centre Neuro-Psycho-Pathologique de l'Université de Kinshasa " C.N.P.P. » en sigle, ont permis de constater que lorsque les symptômes cliniques francs sont stabilisés et qu'ils prennent contact avec la réalité, la plainte principale que la majorité de ces patients présente est l'interaction inadaptée au contexte et une mauvaise adaptation psychosociale. Il s'agit de difficultés que ces patients éprouvent à réagir adéquatement avec le milieu de manière appropriée, à établir un lien affectif adéquat avec les parents et proches, leurs garde-malades, les personnels soignants et d'autres malades partageant en commun le même pavillon, à expliquer leur état morbide et à prendre seuls leur médication. Ces observations ont fait l'objet d'une étude initiale (Tshibwabwa et Mukau, 2018) dans laquelle les comportements d'inadaptation psychosociale des patients observés ont été inventoriés et décrits et les déficits cognitifs évalués en termes des résultats moyens.

Le suivi psychologique en postcure de 24 de ces patients en milieu familial respectif a permis d'observer que malgré une relative absence des symptômes cliniques, la plupart d'entre eux ne participent pas aux activités significatives et signifiantes au quotidien, manifestant entre autres comportements relativement, un défaut d'insight et de awareness (ils n'intègrent pas une image critique d'eux-mêmes), une pauvreté de contact à la réalité (interaction inappropriée), une incapacité de prendre une initiative d'hygiène personnelle (comme se baigner, se brosser les dents, se peigner ou se faire tresser les cheveux, lessiver, repasser et ranger ses habits), complimenter les parents ou les proches, rendre visite aux proches, chercher une information utile, avoir un contrôle sur ses objets d'usage courant, prendre ses médicaments sans aide extérieure, exprimer sa compassion à l'endroit des personnes qui sont dans le moment de détresse ou de malheur dans la famille, etc.

Afin de comparer ces observations avec les constats des personnes témoins des comportements des patients schizophrènes traités, nous avons, à travers un bref 
Tshibwabwa Popi René, Mukau Ebwel Joacim, Yawidi Mayinzambi Jean-Paul, Okitundu Daniel

EVALUATION DU DEVELOPPEMENT COGNITIF ET DES FACTEURS PSYCHOSOCIAUX

DE MAUVAISE ADAPTATION CHEZ LES SCHIZOPHRENES TRAITES AU CENTRE NEURO-PSYCHOPATHOLOGIQUE «C.N.P.P. » DE L'UNIVERSITE DE KINSHASA, DEMOCRATIC REPUBLIC OF THE CONGO

questionnaire d'enquête, recueilli les avis de 31 parents et proches membres de famille qui vivent au quotidien avec les patients sortis d'un traitement au C.N.P.P. sur leurs comportements adaptatifs en famille et dans la communauté ainsi que la possibilité de retour à la vie « normale ». Après analyse de contenu, les personnes interrogées ont réagi comme suit : les patients ont des difficultés de mémoire, par exemple, à se rappeler les détails ou à garder de souvenirs des faits d'un passé proche ou lointain, comme retenir un numéro de téléphone ou encore une adresse d'un proche (89\%), ils manifestent une inattention, particulièrement la difficulté à se concentrer sur une situation, comme écouter attentivement une information à la radio (71\%), ils sont apragmatiques ou incapables à entreprendre une action, par exemple apporter spontanément une aide, prendre soin d'un enfant (85\%), capacités réduites d'exécuter une tâche complexe, par exemple faire un calcul mental, avoir une tolérance à l'effort (69\%), le manque pas d'intérêt pour les activités, par exemple, assister à un culte religieux, compatir au malheur qui frappe la famille (71\%). La quasi-totalité de parents et proches interrogés présentent une inquiétude quant au retour de leurs patients à la vie (normale » notamment la reprise des études, avoir un travail, assumer son statut social. Cette perte relative d'autonomie fonctionnelle ne permet pas à la personne de prendre place dans la famille et dans son environnement.

De la compréhension des comportements d'inadaptation psychosociale dans la schizophrénie, la littérature renseigne que les incapacités fonctionnelles observées chez les personnes souffrant de schizophrénie sont davantage liées aux déficits cognitifs et seraient en grande partie indépendantes des symptômes psychotiques (Comblatt, Green \& Walke, 2007 ; Green, Kern, Braff, \& Mintz, 2000 ; Heinrichs \& Zakzanis, 1998 ; Lewis, 2004 ; Bums \& Patrick, 2007). Selon Favrod et Sheider (2004), environ 85\% des personnes atteintes de schizophrénie souffrent de déficits cognitifs quelle que soit la forme clinique). Grant, Addignton \& Konnert (2001), de leur part, indiquent que presque tous les patients schizophrènes présentent les difficultés cognitives lorsque leur propre fonctionnement pré morbide sert de référence.

Certaines études recensées (Prouteau, 2010 ; Addington \& Gleeson, 2005 ; Roussel, 2006) révèlent que les troubles cognitifs dans la schizophrénie sont présents dès le premier épisode et même avant l'apparition de la maladie. Variant énormément d'un patient à un autre, ils sont relativement stables dans le temps en dehors des périodes de crise ou lorsque les symptômes psychotiques ont disparu, et constituent des marqueurs de trait de la pathologie. Cette thèse est confirmée par des études qui se sont intéressées rétrospectivement au fonctionnement social durant l'enfance et l'adolescence, telles que rapportées par Hans, Auerbach, Asarnow, Styr, \& Marcus, (2000) révélant que les déficits cognitifs liés au fonctionnement social pourraient précéder l'apparition de la maladie. Cette thèse affirme la relation entre les difficultés cognitives et fonctionnement social. Deux revues de la littérature ont également confirmé cette thèse (Green, Kern, Braff, \& Mintz, 2000 ; Green, Kern, \& Heaton, 2004). 
Tshibwabwa Popi René, Mukau Ebwel Joacim, Yawidi Mayinzambi Jean-Paul, Okitundu Daniel

EVALUATION DU DEVELOPPEMENT COGNITIF ET DES FACTEURS PSYCHOSOCIAUX

DE MAUVAISE ADAPTATION CHEZ LES SCHIZOPHRENES TRAITES AU CENTRE NEURO-PSYCHO-

PATHOLOGIQUE «C.N.P.P. » DE L'UNIVERSITE DE KINSHASA, DEMOCRATIC REPUBLIC OF THE CONGO

Selon Franck, 2012 et Franck, 2006), les déficits cognitifs sont réfractaires aux traitements exclusivement médicamenteux, avec des conséquences péjoratives en termes de réinsertion sociale. Diverses études corroborent les auteurs indiquant qu'une diminution marquée des symptômes cliniques après un traitement médicamenteux se traduit rarement en une amélioration significative du niveau de fonctionnement social qui conduit à une meilleure qualité de vie des personnes souffrant de schizophrénie (Gittleman, Klein et Klein, 1969 ; Semkovska, Bédart, Godbout, Limoge, Stip, 2000 ; Green, 1996 ; Cornblatt et al., 2007 ; Green, Kern, Braff, \& Mintz, 2000 ; Robinson, Woemer, McMeniman, Mendelowitz, \& Bilder, 2004).

Les déficits cognitifs sont de plus en plus identifiés comme des facteurs contribuant de façon importante aux difficultés de fonctionnement vécues par une grande partie des personnes souffrant de schizophrénie (McGurk, Moriaty, Harvey, Parrella, White, \& Davis, 2000). Ils interviennent également dans le développement de certains types de symptômes cliniques (délires, hallucinations).

Dans cette optique, l'évaluation des déficits cognitif dans la schizophrénie est une mesure systématiquement reprise pour la mise en place d'une stratégie de remédiation cognitive (D'Amato et Saoud, 2006). A cet effet, un pattern de corrélation entre les déficits cognitifs et les limitations fonctionnelles met en exergue l'altération des fonctions cognitives de base ou l'implication des aspects de la neurocognition (D'Amato et Saoud, 2006 ; Franck, 2006 ; Victor, 2012); Green, et al., 2004). Les traits les plus fréquents touchent la mémoire verbale, l'apprentissage verbal, la flexibilité, la planification, l'inhibition, le raisonnement, la vitesse psychomotrice et l'attention. Prouteau, 2011) souligne l'altération des fonctions cognitives supérieures, intégrant plusieurs fonctions cognitives élémentaires et subissant des influences d'éléments contextuels. Selon Wynes et Spaulding (2011), les patients souffrent également des déficits de la cognition sociale (ensemble des opérations mentales à l'origine des interactions sociales) et de la métacognition (connaissance de ses capacités cognitives et ses contenus de pensée).

Quant à nous, nous nous sommes intéressés à évaluer les fonctions neurocognitives de base de manière à définir les bilans des difficultés cognitives en relation avec la mauvaise adaptation des patients (Robison, Woerner, McMeniman, Mendelowitz \& Bidler, 2004), (Vianin, 2007) car ce sont ces fonctions défaillantes qui devront faire l'objet d'une restauration du processus via les capacités neurocognitives préservées.

Les facteurs psychosociaux qui interfèrent avec leur mauvaise adaptation des patients en sont également évalués. Nous référant à Rapp \& Goscha (1998) et Seligman \& Pawelski (2003), les approches d'évaluation et le traitement des troubles se sont modifiées afin de s'intéresser aux forces et aux intérêts de la personne. Le traitement cherche à s'appuyer sur ce que la personne essaie d'accomplir dans sa vie ainsi que sur ses ressources internes ou externes afin de faciliter son rétablissement. Ainsi, les cibles seront les symptômes, les déficits et les problèmes personnels ou psychosociaux considérées comme des barrières dans la poursuite des buts personnels des patients. Dans ce 
Tshibwabwa Popi René, Mukau Ebwel Joacim, Yawidi Mayinzambi Jean-Paul, Okitundu Daniel

EVALUATION DU DEVELOPPEMENT COGNITIF ET DES FACTEURS PSYCHOSOCIAUX

DE MAUVAISE ADAPTATION CHEZ LES SCHIZOPHRENES TRAITES AU CENTRE NEURO-PSYCHOPATHOLOGIQUE «C.N.P.P. » DE L'UNIVERSITE DE KINSHASA, DEMOCRATIC REPUBLIC OF THE CONGO

rapprochement avec le modèle intégratif "vulnérabilité-stress », nous nous sommes attachés aux aspects relatifs aux stress psychologiques et environnementaux. D'après, Anthony et Liberman (1986), la vulnérabilité génétique, une caractéristique individuelle permanente qui proviendrait d'un héritage génétique et qui réagirait au stress de l'environnement, serait latente et non mesurable directement. Il serait possible de l'évaluer indirectement par ses effets comme résultats de la réadaptation des personnes souffrant de troubles mentaux.

Les facteurs psychologiques évalués aux instruments concernent le coping, l'estime de soi, le rajustement social, la stratégie de traitement et résolution de problèmes. Dans la sphère sociale, sont explorés, à l'aide des outils, le soutien familial perçu et la qualité de relation thérapeutique malade-médecin. D'autres facteurs sont identifiés à l'aide des questions d'entretien clinique ce sont la perception de la maladie (de la personne) par l'entourage familial, l'auto-perception de la maladie par la personne ellemême, la structure de la famille où est issu l'enfant, la consommation des substances et le repérage clinique de la toxicomanie ainsi que l'âge de début de la consommation. Selon le modèle «vulnérabilité-stress-compétence» (Liberman, 2008), ces facteurs sont identifiés comme facteurs de protection susceptibles de favoriser le développement de stratégies adaptatives, d'aider à pallier la vulnérabilité individuelle et influencer les résultats de la réadaptation.

De l'analyse des facteurs de protection, Lysaker \& Buck, \& Dimaggio (2012) indiquent qu'un coping approprié permet de s'adapter à la situation aversive et résoudre le problème. Sur le plan du soutien familial Giron \& Gomez-Beneyto (2004) ont montré qu'il y a une relation entre une empathie des membres de la famille, le fonctionnement occupationnel ainsi que les relations sociales chez les personnes souffrant de schizophrénies. Le soutien social est un facteur de protection nécessaire pour diminuer la résistance au stress et améliorer l'estime de soi (Anthony et Liberman,1986 ; Zubin et Spring (cités par Simonet et Brazzo ,2005). Selon Lambert \& Naber, (2004) et Bourdeau (2012), un support social ainsi qu'une estime de soi positive seraient gage d'une meilleure qualité de vie chez les personnes atteintes de schizophrénie.

Dans la même optique, O’Brien et al. (2008), Howard, Leese, \& Thornicroff (2000) et Giron \& Gomez-Boneyto, (2004) soulignent la relation entre une faible empathie des membres de la famille, le soutien social le niveau faible de fonctionnement social des patients. Les remarques positives des parents étaient associées à une amélioration du fonctionnement des patients souffrant de schizophrénie. A l'inverse, le manque empathie au sein de la famille pourrait être à la base des émotions exprimées, de la stigmatisation et $\mathrm{du}$ rejet des personnes, du fait de l'interprétation du trouble mental en Afrique comme une menace venant de l'extérieur. Il est généralement reconnu que les émotions exprimées au sein de la famille au sein de la famille, par exemple les critiques négatives, le manque de soutien social sont proportionnellement corrélées aux taux de rechute des personnes avec diagnostic de schizophrénie (Turkinton, Mulhollaud, Rush, Anderson, McCaul, Barrett, et al., 2009) ; Butzlaff \& Hooley, 1998). Ces facteurs sont source de stress 
Tshibwabwa Popi René, Mukau Ebwel Joacim, Yawidi Mayinzambi Jean-Paul, Okitundu Daniel

EVALUATION DU DEVELOPPEMENT COGNITIF ET DES FACTEURS PSYCHOSOCIAUX

DE MAUVAISE ADAPTATION CHEZ LES SCHIZOPHRENES TRAITES AU CENTRE NEURO-PSYCHO-

PATHOLOGIQUE «C.N.P.P. » DE L'UNIVERSITE DE KINSHASA, DEMOCRATIC REPUBLIC OF THE CONGO

et interfèrent avec la mauvaise adaptation des patients. A cet effet, Yanos, Lysaker, et Roe, (2010) trouvent que la stigmatisation qui est souvent intériorisée par les personnes atteintes de schizophrénie, a un impact négatif sur leur fonctionnement vocationnel.

Le facteur «relation thérapeutique malade-médecin » est aussi évalué. Autant cette relation est bonne, autant la personne prend conscience de son trouble et la nécessité de prendre sa médication. Les relations thérapeutiques caractérisées par des rapports positifs et facilitants favorisent les bénéfices chez les personnes atteintes de schizophrénie et aurait des répercussions positives sur le fonctionnement social (Hewitt \& Coffey, 2005). Dans une autre perspective, la littérature indique qu'il y a une très forte association entre l'abus de substances et la schizophrénie (Santos, Robert, Riquier et al., 2007). L'abus de SPA est associé à de plus bas niveau de fonctionnement (Menezes et al., 2009 ; Turkinton et al, 2009 ; Butzlaff \& Hooley,1998). L'alcool et le cannabis sont les substances les plus consommées, sans parler bien sûr du tabac. Cette comorbidité serait responsable de certains aspects particuliers du tableau clinique notamment un début plus précoce et plus brutal.

En Afrique noire, la maladie mentale est une expression de rupture des systèmes symboliques qui mettent à mal l'identité du sujet. Elle est interprétée comme une menace externe. La perception de la maladie par les membres de la famille telle que perçue par le patient ainsi que l'auto-perception de la maladie par la personne elle-même influencent les résultats de la réadaptation. La culture propose à l'individu des modèles sur la manière de se sentir et d'agir (Chisholml, \& Bhugra, 1997). Le système social impliquant la famille, l'environnement et la culture influe sur la manière dont la maladie s'exprime, se vit et se traite. L'expérience de la souffrance chez les africains est construite des croyances socioculturelles où le comportement du malade peut être compris et devenir significatif.

\section{Méthodologie}

Nous avons voulu vérifier l'hypothèse selon laquelle: La mauvaise adaptation au contexte que manifeste le patient schizophrène traité au C.N.P.P.de l'Université de Kinshasa serait la conséquence du profil cognitif déficitaire et de la présence des facteurs psychosociaux de mauvaise adaptation associés.

\subsection{Participants}

La population initiale était composée de 48 patients avec diagnostic de schizophrénie traités au C.N.P.P. de l'Université de Kinshasa. Les participants à l'étude ont été constitués de quatre sujets issus de la population de départ sélectionnés par choix raisonné ou de convenance. Partant d'un criterium de contexte notamment le fait que le patient doit être trouvé en sa famille et y résider, être soutenu par sa famille et être suivi par un médecin psychiatre du C.N.P.P., nous nous sommes inspirés des critères de Pirès (1997) pour sélectionner les cas définis comme suit: la pertinence, l'intention 
communicative de l'interlocuteur, la qualité intrinsèque, le bénéfice expérimental à tirer, l'intérêt social, l'accessibilité du cas. Les caractéristiques des cas soumis à l'étude sont présentées dans le tableau ci-dessous.

Table 1 : Caractéristiques identificatoires des participants

\begin{tabular}{|l|l|l|l|c|c|c|}
\hline \multirow{2}{*}{ Sujet } & \multirow{2}{*}{ Sexe } & Diagnostic & \multicolumn{2}{|c|}{ Age } & \multicolumn{2}{c|}{ Autres renseignements } \\
\cline { 4 - 7 } & & & $\mathbf{1}^{\text {ère }}$ crise & Réel & $\begin{array}{c}\text { Etat-Civil / } \\
\text { Nombre d'enfants }\end{array}$ & $\begin{array}{c}\text { Etudes } \\
\text { faites }\end{array}$ \\
\hline Cas 1 & Masculin & Schizo-affectif & 23 ans & 33 ans & Célibataire & 3ème Secondaire \\
\hline Cas 2 & Féminin & Schizo-affectif & 26 ans & 47 ans & Marié +6 & Diplômé d'état \\
\hline Cas 3 & Masculin & Paranoïde & 17 ans & 23 ans & Célibataire & Diplômé d'état \\
\hline Cas 4 & Masculin & Schizophréniforme & 25 ans & 47 ans & Célibataire +1 & Non scolarisé \\
\hline
\end{tabular}

\subsection{Instruments de mesure utilisés :}

- Pour le bilan neurocognitif : la figure complexe de Rey et le Mini Mental State Examination- MMSE (version française consensuelle).

- Pour le bilan des facteurs psychologiques

\subsubsection{Echelles de mesure sociale}

Le WCC-R de Vitaliano et al., 1985 (coping d'ajustement ou la manière dont la personne s'adapte à sa situation aversive), Estime de soi de Rosenberg (jugement et évaluation de la valeur personnelle), Rajustement social de Rahe et Holmès (facilité et fréquence avec lesquelles le sujet est affecté par les événements stressants) ;

\subsubsection{Test psychologique}

Les Cubes de Kohs (stratégie de résolution et de traitement de l'information adoptée par la personne).

\subsection{Pour le bilan des facteurs sociaux}

\subsubsection{Instruments}

Questionnaire de soutien social perçu (QSSP) de Brochon-Schweitzer (2001) (le sujet perçoit-il le soutien de sa famille ?), Echelle du vécu de la relation thérapeutique(ERT) de Cottraux (quelle est la qualité de la relation malade-médecin traitant?)

\subsubsection{Entretien clinique}

Perception de la maladie par l'entourage familial (comment vos parents et vos proches interprètent-ils votre maladie ?) ; Auto-perception de la maladie (comment expliquezvous et comprenez votre maladie ?) ; Structure familiale où est issue l'enfant (quelle est la structure de votre famille?); Consommation des substances psychoactives (consommez-vous des substances ? Si oui, lesquelles ? Fréquence ? Quantité ? Age de début de la consommation? Repérage clinique de la toxicomanie. 


\section{Résultats}

\subsection{Bilans neurocognitifs (atteintes neurocognitives)}

N.B. Les tests neurocognitifs sont intellectuels et sensibles aux facteurs culturels. Le patient cas 4 non scolarisé n'est pas soumis à l'évaluation du processus cognitif.

\section{Cas 1}

1. Figure complexe de Rey (cotation qualitative essentiellement) :

Les résultats ont révélé :

- L'absence de 6 éléments de la copie (déficit de la mémoire visuelle);

- Une reproduction 2 par détails retardée et inachevée (déficits de planification) ;

- Une fragmentation retardée (déficit d'attention-concentration) ;

- Une passivité dans le rappel immédiat d'une figure 3' (déficit de la mémoire immédiate (du travail) et de la vitesse d'exécution);

- Une ressemblance fausse (déficit de la mémoire immédiate) ;

- Une fausse reconnaissance d'une figure (déficit de l'attention et de la mémoire à long terme) ;

- Vitesse d'exécution 323"'/900 (impulsivité).

2. Mini Mental State Examination (MMSE)

Les résultats ont révélé :

- Une préservation relative de la mémoire épisodique temporelle (score : 3/5)

- Un déficit léger de la mémoire épisodique spatiale (score : $2 / 5$ ) ;

- Déficit sévère de la mémoire immédiate (instantanée) de rappel (score $1 / 5$ ) ;

- Déficit de l'attention-calcul 'exécution) (score 2/5) ;

- Déficit de la mémoire de rappel différé (à long terme) (score : 1/3) ;

- Une préservation relative de construction langage (score $1 / 2$ ) ;

- Déficit de la mémoire à long terme (score 0/1) ;

- déficit d'exécution-construction (score1/3).

\section{Cas 2}

1. Figure complexe de Rey (cotation qualitative)

Les résultats à la Figure complexe de Rey ont révélé :

- L'absence de plusieurs d'éléments de la copie (déficit sévère de la mémoire visuelle) ;

- Une copie du modèle 3 inachevée (déficit planification et stratégie de résolution de problèmes);

- Une hésitation à fragmenter la figure (déficit d'attention-construction) ;

- Une fausse ressemblance au rappel immédiat (déficit de la mémoire du travail) ;

- Une mauvaise reconnaissance de la figure (déficit de mémoire à long terme)

- Vitesse d'exécution : 301"/900, Vitesse de rappel : 287', vitesse de reconnaissance : 255" (précipitation et impulsivité) ;

- Exécution de la copie inachevée (déficit d'attention visuo-construction). 
2. Mini Mental State Examination (MMSE)

Les résultats du MMSE ont révélé :

- Une mémoire épisodique temporelle déficitaire (score : $2 / 5$ )

- Un déficit de la mémoire épisodique spatiale (score : $2 / 5$ )

- Un déficit de la mémoire de rappel immédiat (instantanée) (score : 1/3)

- Un déficit de l'exécution (attention et calcul (score :2/5) ;

- Rappel différé des mots (mémoire à long terme déficitaire) (score1/3)

- Déficits sévères de praxies constructives (score :0).

\section{Cas 3}

1. Figure de Rey

- Choix du plan 1 : mémoire visuelle-relative préservation

- Plan 3 et difficile commencement : déficit de planification ;

- Fragmentation lente : attention-concentration-déficit léger ;

- Rappel immédiat lent : déficit léger mémoire de travail ;

- Rappel différé 2 formes incomplètes-déficit mémoire à long terme ;

- Reconnaissance partielle : déficit léger de la mémoire différée ;

- Attention visuo-construction préservation relative ;

\section{MMSE}

- Mémoire épisodique temporelle 3/5 préservation ;

- Mémoire épisodique spatiale 3/5 préservation ;

- Rappel immédiat $1 / 3$ mémoire instantanée déficitaire ;

- Attention-calcul (exécution) 2/5 déficit attention-exécution ;

- Rappel différé de mots :1/3 déficit de la mémoire à long terme ;

- langage : 4/8 capacité de constriction langagière-moyenne ;

- Praxies-construction : 1/1 préservation de la capacité d'exécution des tâches.

\subsection{Bilan des facteurs psychologiques}

Table 2 : Coping et stratégie d'ajustement au stress

\begin{tabular}{|l|l|}
\hline Cas 1 & Coping centré sur le soutien \\
\hline Cas 2 & Coping émotionnel \\
\hline Cas 3 & Coping centré sur le soutien \\
\hline Cas 4 & Coping émotionnel \\
\hline
\end{tabular}

Les résultats du coping révèlent que les sujets adoptent les stratégies d'ajustement inappropriées (émotionnel et de soutien) 
Tshibwabwa Popi René, Mukau Ebwel Joacim, Yawidi Mayinzambi Jean-Paul, Okitundu Daniel

EVALUATION DU DEVELOPPEMENT COGNITIF ET DES FACTEURS PSYCHOSOCIAUX

DE MAUVAISE ADAPTATION CHEZ LES SCHIZOPHRENES TRAITES AU CENTRE NEURO-PSYCHO-

PATHOLOGIQUE «C.N.P.P. » DE L'UNIVERSITE DE KINSHASA, DEMOCRATIC REPUBLIC OF THE CONGO

Table 3 : Estime de soi

\begin{tabular}{|l|c|c|}
\hline Sujets & Scores & Niveau de l'estime de soi \\
\hline Cas1 & $($ ) $25-31$ & Faible \\
\hline Cas 2 & $<25$ & Très faible \\
\hline Cas 3 & $($ ) $31-34$ & Moyenne \\
\hline Cas 4 & $<25$ & Très faible \\
\hline
\end{tabular}

Les résultats montrent que les sujets ont une estime de soi faible.

Table 4 : Rajustement social

\begin{tabular}{|l|c|c|}
\hline Sujet & Scores & Rajustement social \\
\hline Cas 1 & $200-299$ & stress élevé \\
\hline Cas 2 & 299 & stress très élevé \\
\hline Cas 3 & $150-199$ & stress modéré \\
\hline Cas 4 & $200-299$ & stress élevé \\
\hline
\end{tabular}

Les sujets manifestent une sensibilité au stress élevée.

Table 5 : Stratégie de traitement et résolution de problèmes adoptées

\begin{tabular}{|l|c|}
\hline Sujet & Stratégie adoptée \\
\hline Cas 1 & Globale (par gestalt) \\
\hline Cas 2 & Globale \\
\hline Cas 3 & Globo-analytique \\
\hline Cas 4 & Global \\
\hline
\end{tabular}

Les patients abordent les problèmes par la stratégie globale qu'analytique

\subsection{Bilan des facteurs sociaux}

Table 6 : Soutien social perçu

\begin{tabular}{|l|c|}
\hline Sujet & Soutien social \\
\hline Cas 1 & Non perçu \\
\hline Cas 2 & Non perçu \\
\hline Cas 3 & Relativement perçu \\
\hline Cas 4 & Non perçu \\
\hline
\end{tabular}

Les sujets ne perçoivent le soutien familia.

Table 7 : Qualité de la relation thérapeutique malade-médecin

\begin{tabular}{|l|c|}
\hline Sujet & Qualité de la relation thérapeutique \\
\hline Cas 1 & Score ( ) 12-31 Moins bonne \\
\hline Cas 2 & Score ( ) 52-72 Médiocre \\
\hline Cas 3 & Score ( ) 12-31 Relativement bonne \\
\hline Cas 4 & Score ( ) 32-51 Moins bonne \\
\hline
\end{tabular}


Tshibwabwa Popi René, Mukau Ebwel Joacim, Yawidi Mayinzambi Jean-Paul, Okitundu Daniel

EVALUATION DU DEVELOPPEMENT COGNITIF ET DES FACTEURS PSYCHOSOCIAUX

DE MAUVAISE ADAPTATION CHEZ LES SCHIZOPHRENES TRAITES AU CENTRE NEURO-PSYCHO-

PATHOLOGIQUE «C.N.P.P. » DE L'UNIVERSITE DE KINSHASA, DEMOCRATIC REPUBLIC OF THE CONGO

Le vécu de la relation thérapeutique malade-médecin est moins bonne

Table 8 : Perception de la maladie par l'entourage :

Comment les autres, parents et proches interprètent-ils votre maladie?

\begin{tabular}{|l|c|}
\hline Sujet & Perception de la maladie \\
\hline Cas 1 & Sorcellerie, mauvais sort, famille \\
\hline Cas 2 & Sorcellerie, jalousie \\
\hline Cas 3 & Jalousie, envoutement \\
\hline Cas 4 & Esprit diabolique, magie \\
\hline
\end{tabular}

Les parents et proches des patients attribuent la maladie aux causes externes.

Table 9 : Auto-perception de la maladie :

Comment comprenez-vous et expliquez votre maladie ?

\begin{tabular}{|l|c|}
\hline Sujet & Auto-perception de la maladie \\
\hline Cas 1 & Punition de Dieu, soucis \\
\hline Cas 2 & Famille, jalousie \\
\hline Cas 3 & Jalousie \\
\hline Cas 4 & Diable, maladie mystique \\
\hline
\end{tabular}

Les résultats indiquent le sujet perçoit sa maladie comme une menace, une attaque extérieure.

Table 10 : Structure familiale où est issu l'enfant : Quelle est la structure de votre famille?

\begin{tabular}{|l|c|}
\hline Sujets & Structure familiale \\
\hline Cas 1 & Nucléaire \\
\hline Cas 2 & Nucléaire \\
\hline Cas 3 & Recomposition maternelle \\
\hline Cas 4 & Polygamique \\
\hline
\end{tabular}

Les sujets sont, également, issus de la structure familiale nucléaires que désunies (à recomposition et polygamique non sécures).

Table 11 : Consommation des substances psychoactives :

Consommez-vous des substances? Si oui, lesquelles?

\begin{tabular}{|l|c|}
\hline Sujets & Types de Substances consommées \\
\hline Cas 1 & Cannabis, tabac, alcool fort \\
\hline Cas 2 & - \\
\hline Cas 3 & Cannabis, tumbacco \\
\hline Cas 4 & Cannabis, alcool fort \\
\hline
\end{tabular}

Les résultats révèlent que les sujets consomment les substances, surtout le cannabis et l'alcool. 
Tshibwabwa Popi René, Mukau Ebwel Joacim, Yawidi Mayinzambi Jean-Paul, Okitundu Daniel

EVALUATION DU DEVELOPPEMENT COGNITIF ET DES FACTEURS PSYCHOSOCIAUX

DE MAUVAISE ADAPTATION CHEZ LES SCHIZOPHRENES TRAITES AU CENTRE NEURO-PSYCHO-

PATHOLOGIQUE «C.N.P.P. » DE L'UNIVERSITE DE KINSHASA, DEMOCRATIC REPUBLIC OF THE CONGO

Table 12 : Fréquences de consommation des substances psychoactives :

Si oui, quelle est la fréquence?

\begin{tabular}{|l|c|}
\hline Sujets & Fréquence de consommation des substances \\
\hline Cas 1 & Régulière \\
\hline Cas 2 & - \\
\hline Cas 3 & Régulière \\
\hline Cas 4 & Quotidienne \\
\hline
\end{tabular}

Les résultats montrent une consommation régulière et quotidienne des substances psychoactives.

Table 13 : Si oui, quelle est la quantité ?

\begin{tabular}{|l|c|}
\hline Sujets & $\begin{array}{c}\text { Quantité des substances } \\
\text { psychoactives consommées }\end{array}$ \\
\hline Cas 1 & Excès fréquent \\
\hline Cas 2 & - \\
\hline Cas 3 & Excès fréquent \\
\hline Cas 4 & Toujours avec excès \\
\hline
\end{tabular}

Les sujets consomment les substances en quantité excessive et fréquente.

Table 14 : Si oui, quel est le mode de consommation que vous utilisé ?

\begin{tabular}{|l|c|}
\hline Sujets & $\begin{array}{c}\text { Mode de consommation } \\
\text { des substances psychoactives }\end{array}$ \\
\hline Cas 1 & Oral, fumée \\
\hline Cas 2 & - \\
\hline Cas 3 & Oral, narine \\
\hline Cas 4 & Oral, fumée \\
\hline
\end{tabular}

Les modes de consommation des substances utilisée par les patients son oral et par la fumée.

Table 15 : A quel âge avez-vous débuté à consommer les substances psychoactives ?

\begin{tabular}{|l|c|}
\hline Sujets & $\begin{array}{c}\text { Début de consommation } \\
\text { des substances psychoactives }\end{array}$ \\
\hline Cas 1 & 13 ans \\
\hline Cas 2 & - \\
\hline Cas 3 & 17 ans \\
\hline Cas 4 & 14 ans \\
\hline
\end{tabular}

Les sujets ont commencé à consommer les substances à l'adolescence et même avant cette périod. 
Tshibwabwa Popi René, Mukau Ebwel Joacim, Yawidi Mayinzambi Jean-Paul, Okitundu Daniel

EVALUATION DU DEVELOPPEMENT COGNITIF ET DES FACTEURS PSYCHOSOCIAUX

DE MAUVAISE ADAPTATION CHEZ LES SCHIZOPHRENES TRAITES AU CENTRE NEURO-PSYCHOPATHOLOGIQUE « C.N.P.P. » DE L'UNIVERSITE DE KINSHASA, DEMOCRATIC REPUBLIC OF THE CONGO

Table 16 : Repérage clinique de la toxicomanie

\begin{tabular}{|l|c|}
\hline Sujets & Repérage clinique de la toxicomanie \\
\hline Cas 1 & Dépendance \\
\hline Cas 2 & - \\
\hline Cas 3 & Usage nocif \\
\hline Cas 4 & Dépendance \\
\hline
\end{tabular}

Les patients ont une consommation nocive et dépendante des substances psychoactives

\section{Discussion}

Les résultats mettent en évidence des déficits cognitifs variables et des facteurs psychologiques et sociaux associés qui interfèrent avec la mauvaise adaptation chez tous les patients. La discussion fait l'interprétation des résultats, les compare entre eux et avec la littérature.

\subsection{Bilans cognitifs}

Les tests cognitifs utilisés explorent les fonctions cognitives au regard du pattern ou gardent de sévérité de corrélation entre les déficits cognitifs et les limitations fonctionnelles dans la schizophrénie qui implique l'atteinte des fonctions neurocognitives de base. Les tâches de la vie sociale, professionnelle et indépendante exigeant la capacité à apprendre de nouvelles informations (Franck, 2006 ; Victor, 2012 ; Green, et al., 2004 ; Roussel, 2006), la relation serait très forte avec la mémoire déclarative. Les résultats des bilans neurocognitifs réalisés à l'aide de la Figure complexe de Rey et de MMSE ont révélé des déficits variables des fonctions cognitives de base chez tous les patients, avec une préservation relative des certaines fonctions, sur le plan individuel. La planification des tâches, la mémoire immédiate instantanée), la mémoire de reconnaissance et l'attention exécution se sont montrées déficitaires chez tous les sujets. La mémoire épisodique temporelle est relativement préservée. Ces résultats des bilans neurocognitifs corroborent l'étude de Roussel (2006) qui indique une forte relation entre les déficits cognitifs et la mémoire, et rejoignent ceux de l'étude menée par Morice et Delahunty dans laquelle $94 \%$ des patients suivis en externe avaient un déficit au moins dans un des trois domaines des fonctions exécutives et la mémoire de travail (cités par Roussel, 2006).

\subsection{Bilans des facteurs psychologiques}

Les bilans des profils psychologiques individuels ont révélé, de façon variable, des facteurs psychologiques de mauvaise adaptation psychosociale chez tous les sujets. En effet, faire face à leur situation, les patients utilisent un coping inapproprié, soit d'évitement, soit de soutien. Ils ne mettent pas en place les stratégies adaptatives (coping) permettant de surmonter des situations stressantes source de l'inadaptation sociale (Lysaker, Erickson, Buck \& Dimaggio, 2012 ; Anthony et Liberman,1986). 
Tshibwabwa Popi René, Mukau Ebwel Joacim, Yawidi Mayinzambi Jean-Paul, Okitundu Daniel

EVALUATION DU DEVELOPPEMENT COGNITIF ET DES FACTEURS PSYCHOSOCIAUX

DE MAUVAISE ADAPTATION CHEZ LES SCHIZOPHRENES TRAITES AU CENTRE NEURO-PSYCHO-

PATHOLOGIQUE «C.N.P.P. » DE L'UNIVERSITE DE KINSHASA, DEMOCRATIC REPUBLIC OF THE CONGO

Les patients ont manifesté à l'évaluation une estime de soi est faible. Ils sont moins portés à se fixer des buts. Ce facteur est une cible importante de la réadaptation des patients souffrant de schizophrénie (Bourdeau (2012).

L'évaluation du rajustement social a révélé que les patients ont une sensibilité élevée au stress de l'environnement. Ces résultats corroborent Anthony et Liberman (1986) qui ont montré que le stress, suscité par tant par les ennuis que par les événements importants de la vie ou la présence d'émotions exprimées dans l'environnement, peut augmenter les détériorations, les déficits et les handicaps associés à la psychose.

Aux tâches des cubes de Kohs, les sujets ont tendance à résoudre les problèmes par des stratégies globales. Ils superposent les cubes de façon hâtive sans envisager d'analyser les situations par dissection.

\subsection{Bilans et des facteurs sociaux}

Les patients ont présenté des facteurs sociaux de mauvaise adaptation à l'environnement parmi lequels le soutien social. Les patients ne perçoivent pas le soutien de la famille. Par cette carence, ils sentiraient stigmatisés. Ces résultats rencontrent Lambert \& Naber (2004) et Yanos, Lysaker, et Roe, (2010) qui trouvé d'une part que la stigmatisation est souvent intériorisée par les personnes atteintes de schizophrénie et d'autre part, le support social ainsi qu'une estime de soi positive seraient gage d'une meilleure qualité de vie chez les personnes atteintes de schizophrénie. Un patient non soutenu diminuera le sentiment d'auto-détermination et sa motivation aux activités.

Tous les sujets ne sont pas en bonne relation « malade-médecin ». Il manque d'une complicité thérapeutique ne favorise la compliance et l'adhésion au traitement. Les entretiens cliniques ont révélé les habitudes de consommation régulière, avec excès et dépendance, de cannabis et l'alcool fort sont observées chez les sujets masculins. La consommation de substances constitue un facteur de rechute. Une étude de Scheier et al (2000) rapporte que la consommation de l'alcool entraîne les rechutes, source de la dépression et stress chez les personnes souffrant de psychoses ainsi qu'un faible niveau des habiletés et compétences sociales. La consommation de drogue est associée aux à un faible niveau d'habiletés sociales et prédictive de l'adaptation sociale.

Les sujets interprètent leur maladie comme une menace extérieure. Cette façon d'interpréter le trouble ne favoriserait pas l'insight et le awareness. Elle ne favorise non plus l'alliance thérapeutique.

Les proches ont la même perception de la maladie. Cette attribution causale de la maladie est source des confits en famille.

Les patients sont issus également des familles désunies, polygamiques ou à recomposition. Ces structures familiales ne sont pas sécures pour le développement affectif des enfants. 


\section{Conclusion et recommandations}

\subsection{Conclusion}

Cette étude clinique s'était proposée d'évaluer, à l'aide des tests neuropsychologiques, échelles de mesure sociale et entretiens, les déficits cognitifs et les facteurs psychologiques et sociaux de mauvais adaptation qui interfèrent avec la mauvaise adaptation chez quatre patients schizophrènes traités au Centre Neuro-PsychoPathologique «C.N.P.P. » de Les bilans individuels ont révélé des déficits variés des fonctions cognitives de base dans les domaines de la mémoire, de l'attention et des fonctions exécutives, ainsi que la présence des facteurs psychologiques et environnementaux de mauvaise adaptation.

Les troubles cognitifs constituent une des caractéristiques centrales de la schizophrénie. Selon Heinrichs \& Zakzanis (1998), ils sont présents dès le premier épisode, stables et persistent longtemps chez le patient même lorsque les symptômes psychotiques sont stabilisés. La stabilité persistante dans le temps de ces déficits cognitifs associés aux facteurs psychologiques et sociaux occasionne le déficit du fonctionnement et la perte des habiletés sociales nécessaire à la participation aux activités significatives et signifiantes de la vie quotidienne.

Par ailleurs, les études révèlent qu'une diminution marquée des symptômes cliniques après un traitement médicamenteux se traduit rarement en une amélioration significative du fonctionnement social. (Cornblatt et al., 2007 ; Green, Kern, Braff, \& Mintz, 2000). Dans cette perspective, le recours à une approche cognitive et aux interventions de réadaptation psychosociale individualisée permettraient de restaurer le processus cognitif et de favoriser la participation aux activités quotidiennes, le retour à la famille et à la communauté, au rythme individuel.

\subsection{Recommandation}

Au regard des résultats obtenus qui ont révélé des déficits cognitifs et des facteurs de mauvaise adaptation associés, un programme de remédiation cognitive adaptée au contexte allié aux interventions individualisées de réadaptation sociale permettent d'améliorer le statut cognitif, le fonctionnement et la qualité de vie des patients (Gittleman, Klein et Klein, 1969 ; Cornblatt et al., 2007 ; Green, Kern, Braff, \& Mintz, 2000), au rythme individuel.

\section{Bibliographie}

Addington, J. \& Gleeson, J. (2005). Implementing cognitive-behavioral therapy for firstepisode psychosis. British Journal of Psychiatry-Supplementum, 48,572-76.

Amiel-Lebigre, F. (1996). Evénements stressants de la vie : méthodologies et résultats. Encyclopédie médico-chirurgicale, 37, 401, E1. 
Tshibwabwa Popi René, Mukau Ebwel Joacim, Yawidi Mayinzambi Jean-Paul, Okitundu Daniel

EVALUATION DU DEVELOPPEMENT COGNITIF ET DES FACTEURS PSYCHOSOCIAUX

DE MAUVAISE ADAPTATION CHEZ LES SCHIZOPHRENES TRAITES AU CENTRE NEURO-PSYCHO-

PATHOLOGIQUE « C.N.P.P. » DE L'UNIVERSITE DE KINSHASA, DEMOCRATIC REPUBLIC OF THE CONGO

Anthony, W. A. \& Liberman, R. P. (1986). The practice of psychiatric rehabilitation: Historical, conceptual, and research base. Schizophrenia Bulletin, 12, 542-559.

Beck, F. S. Legleye, P., \& Peretti-Wattel. (2006). Drogues \& dépendance, le livre d'information, édition INPES, Avr 2006 : 106-117.

Beuscart-Zéphir, M-C., Anceaux F., Duhamel, A. \& Quentin, S. (1996). Un exemple d'application du tic cognitif, Psychologie Française, 41, 1, 65-76.

Bourdeau, G. (2012). Fonctionnement social et rétablissement à la suite d'un premier épisode psychotique. Thèse de doctorat en psychologie clinique. Université de Montréal.

Burns, T. \& Patrick, D. (2007). Social functioning as an outcome measure in schizophrenia studies. Acta Psychiatric Scandinavica, 116, 403-418.

Butzlaff, R. L., \& Hooley, J. M. (1998). Expressed emotion and psychiatric relapse : a metaanalysis. Archive of General Psychiatry, 55, 547-552.

Butzlaff, R. L. \& Burns, J., Long, J. \& Kay, D. D. (2007). The structure and invariance of model of social functioning in schizophrenia. The Journal of Nervous and Mental Disease. 190(2), 63-72.

Chapman, L. J., \& Chapman, J. P. (1989). Stratégies for resolving the heterogeity of schizophrenics and their relatives using cognitive measures. Journal of Abnormal Psychology, 98(4), 357-366.

Chisholml, D. \& Bhugra, D. (1997). Sociocultural and economic aspects of quality of life measurement. Eur Psychiatry (12), 210-215.

Cornblatt, B., Lencz, T., Smith, C., Olsen R., Auther, A., Nakayama, E., et al. (2007). Can antidepressants be used to treat the schizophrenia prodrome? Results of a prospective, naturaliste treatment study of adolescents. Journal of Clinical Psychiatry, 68(4),546-557.

D’Amato, T. et Saoud, D. (2006). La schizophrénie de l'adulte. De causes aux traitements, Paris : Masson.

Favrod, J. \& ScheIder, D. (2004). Se rétablir de la schizophrénie: un modèle d'intervention. In Rev Med Suisse Romande ; 124 : 205-8.

Franck, N. (2012). Métacognition et théorie de l'esprit dans la schizophrénie. In : Remédiation cognitive. Issy les Moulineaux : Masson.

Franck, N. (2006). La schizophrénie : La reconnaître et la soigner, Paris : Odile Jacob.

Friard, D., et Lavoine P-L. (2004). schiz'ose dire, n5, « La schizophrénie, ça se soigne » édition Lilly Presse, Mar 2004.

Giron, M., \& Gomez-Beneyto, M. (2004). Relationship between Family Attitudes and Social Functioning in Schizophrenia : A Nine-Month Follow-Up Prospective Study in Spain. The Journal of Nervous and Mental Disease, 192(6), 414-420.

Grant, C., Addington, J., Addington, D., \& Konnert, C. (2001). Social functioning in first and multiepisode schizophrenia. Canadian Journal of psychiatriy - Revue Canadienne de Psychiatrie, 46(8), 746-748. 
Tshibwabwa Popi René, Mukau Ebwel Joacim, Yawidi Mayinzambi Jean-Paul, Okitundu Daniel

EVALUATION DU DEVELOPPEMENT COGNITIF ET DES FACTEURS PSYCHOSOCIAUX

DE MAUVAISE ADAPTATION CHEZ LES SCHIZOPHRENES TRAITES AU CENTRE NEURO-PSYCHO-

PATHOLOGIQUE « C.N.P.P. » DE L'UNIVERSITE DE KINSHASA, DEMOCRATIC REPUBLIC OF THE CONGO

Green, M., Kern, R., Braff, D., \& Mintz, J. (2000). Neurocognitive deficits and functional outcome in schizophrenia: are we measuring the "right stuff"? Schizophrenia Bulletin, 26 (1), 119-136.

Green, M., Kern, R. \& Healton, R. K. (2004). Longitudinal studies of cognition and functional outcome in schizophrenia: implications for MATRICS. Schizophrenia Research, 72(1), 41-51.

Hans, S.L., Auerbach, J.G., Asarnow, J.R., Styr, B. \& Marcus, J. (2000). Social adjustment adolescents as risk for schizophrenia : the Jerusalem Infant Development Study. Journal of the American Academy of Child \& Adolescent Psychiatry, 39(1), 1406-1414.

Heinrichs, R. W. \& Zakzanis, K. K. (1998). Neurocognitive deficit in schizophrenia: a quantitative review of the evidence. Neuropsychology ; 12 : 426-45.

Hewitt, J. \& Coffey, M. (2005). Therapeutic working relationships with people with schizophrenia : littérature review. Journal Avanced Nursing, 52(5), 561-570.

Howard, L., Leese, M., \& Thornicroft, G. (2000). Social networks and functioning status in patients with psychosis. Acta psychiatrica Scandinavica, 102(5), 376-385.

Knight, J. A., Kapland, E., \& Ireland, L. D. (2003). Survey findings of Rey Osterrieth Complex Figure usage. In J. A. Knight \& E. F. Kaplan (Eds.), Handbook of ReyOsterrieth Complex Figure Usage : Clinical and research applications (pp. 45-56). Odessa, FL: Psychological Assessment Resources.

Lewis, R. (2004). Should cognitive deficit be a diagnostic criterion for schizophrenia? Journal of psychiatry $\mathcal{E}$ neuroscience, 29, 102-113.

Liberman, R.P. (2008). Recovery from disability-Manuel of psychiatric rehabilitation. Arlington, VA : American Psychiatric Publishing, Inc.

Lysaker, P., Erickson, M., Buck, KD., Dimaggio, G. (2012). Les rapports entre métacognition, cognition et fonctionnement dans la schizophrénie : données issues de l'étude de récits personnels. Inf Psychiatr;88: 267-77.

Lysaker, P., Erickson, M., Buck, KD., Dimaggio, G. (2012). Les rapports entre métacognition, cognition et fonctionnement dans la schizophrénie : données issues de l'étude de récits personnels. Inf Psychiatr;88: 267-77.

McGurk S. R., Meltzer H. Y. (2000). The role of cognition in vocational functioning in schizophrenia. Schizophr Res 2000; 45:175-84.

McGurk, S. R., Moriaty, P. J., Harvey, P. D., Parrella, M., White, L., \& Davis, K. L. (2000). The longitudinal relationship of clinical symptoms, cognitive functioni,g, and adaptive life in geriatric schizophrenia. Schizophrenia Research, 41(1), 47-55.

Menezes, N. Malla, A., Norman, R., Archie, S., Roy, P., \& Zipursky, R. (2009). A mulitisite Canadian perspective. Examination the functional outcome from first-episode psychosis. Acta Psychiatrica Scandinavica, 120(2),138-146.

O’Brien, M., Zinberg, J. L., Bearden, C., Lopez, S., Kopelowicz, A., Daley, M. et al. (2008). Parent attitudes and parent adolescent interaction in families of youth at risk for psychosis and with recent-onset psychotic symptoms. Early Intervention in Psychiatry, 2(4), 268-276. 
Tshibwabwa Popi René, Mukau Ebwel Joacim, Yawidi Mayinzambi Jean-Paul, Okitundu Daniel

EVALUATION DU DEVELOPPEMENT COGNITIF ET DES FACTEURS PSYCHOSOCIAUX

DE MAUVAISE ADAPTATION CHEZ LES SCHIZOPHRENES TRAITES AU CENTRE NEURO-PSYCHO-

PATHOLOGIQUE « C.N.P.P. » DE L'UNIVERSITE DE KINSHASA, DEMOCRATIC REPUBLIC OF THE CONGO

Osterrieth, P. A. (1944). Le test de copie d'une figure complexe ; contribution à l'étude de la perception et de la mémoire. [Test of copying a complex figure ; contribution to the study of perception and memory.]. Archives de Psychologie, 30, 206-356.

Pirès, A. (1997). Echantillonnage et recherche qualitative : essai théorique et méthodologique. Dans J. Poupart, J., Deslauriers, L., Groulx, A. Laperrière, P. Mayer \& A. Pirès, La recherche qualitative : Enjeux épistémologiques et méthodologiques, 19 (p.113-172), Boucherville : G. Morin.

Prouteau, A. (2011). Neuropsychologie clinique de la schizophrénie. Dunod.

Rapp, C. A., \& Goscha, R. J. (1998). The strengths Model: Case management with Pychiatric Disabilities.(2nd Edition). New York : The Oxford University press.

Rey, A. (1941). Test de copie et de reproduction de mémoire de figures géométriques complexes. Paris : ECPA.

Robison, D. G., Woerner, M. G., McMeniman, M., Mendelowitz, A. \& Bilder, R. M. (2004). Symptomatic and functional recovery from a first episode of schizophrenia or schizoaffective disorder. American Journal of Psychiatry, 161(3), 474-479.

Roussel, C. (2006). Le processus de réhabilitation des patients atteints de schizophrénie : Place et enjeux de la remédiation cognitive. Thèse de doctorat en médecine. Université Joseph Fourier, Grenoble.

Saoud, M. et d'Amato, T. (2006). La schizophrénie de l'adulte, édition Masson, 2006 : 117.

Santos, P., Robert, P., Riquier, G., et al. (2007). Santé mentale, une passerelle pour la rémission, édition Janssen-Cilag, Jan 2007 : 51-54.

Seligan, M., \& Pawelski, J.O. (2003). Positive psychology : FAQs. Psychological Inquiry, 14, 159-163.

Simonet, M. \& Brazo, P. (2005). Modèle cognitivo-comportemental de la schizophrénie. EMC (Elsevier Masson SAS, Paris,), Psychiatrie, 37-290-A-10.

Tshibwabwa Popi et Mukau Ebwel (2018). Bilan des déficits cognitifs des patients schizophrènes traités au Centre Neuro-Psycho-Pathologique « C.N.P.P. » de 1'Université de Kinshasa ; In CRIDUPN, nº 076a Juillet-Septembre 2018, 232-248.

Turkinton, A., Mulhollaud, C. C., Rush, T. M., Anderson, R., McCaul, R., Barrett, S. L., et al. (2009). Impact of persistent substance measure on 1-year outcome in first episode psychosis. British Journal of Psychiatry, 95(3), 242-248.

Tupler, L. A., Welsh, K. A., Asare-Aboagye, Y., \& Dawson, D. V. (1995). Reliability of the Rey Osterrieth Complex Figure in use with memory impaired patients. Journal of Clinical and Experimental Neuropsychology, 17(4), 566-579. doi: 10.1080/01688639508405 146

Vianin, P. (2007). Remédiation cognitive de la schizophrénie. Présentation du programme RECOS. Annales Médico Psychologiques ; 165 : 200-205.

Vitaliano, P., Russo, J., Carr, JE., Maiuro, R.D., \& Becker, J. (1985). The ways of coping checklist: Revision and psychometric properties, Multivariate Behavioral Research, $20,3,-26$. 
Wechsler, D. (1956). La mesure de l'intelligence de l'adulte, Paris : PUF.

Wykes, T. \& Spaulgdng, W. D. (2011). Thinking about the future cognitive remediation Therapy-What works and could we do better? Schizophrenia Bulletin,37(2), 80.90.DOI :10.1093/schbul/sbr064

Yanos, P., Lysaker, P. H., \& Roe, D. (2010). Internalized stigma as a barrier to improvement in vocational functioning among with schizophrenia-spectrum disorders. Psychiatriy Research, 17(1), 211-213. will be applied to their work. Under the terms of this license, no permission is required from the author(s) or publisher for members of the community to copy, distribute, transmit or adapt the article content, providing a proper, prominent and unambiguous attribution to the authors in a manner that makes clear that the materials are being reused under permission of a Creative Commons License. Views, opinions and conclusions expressed in this research article are views, opinions and conclusions of the author(s). Open Access Publishing Group and European Journal of Social Sciences Studies shall not be responsible or answerable for any loss, damage or liability caused in relation to/arising out of conflicts of interest, copyright violations and inappropriate or inaccurate use of any kind content related or integrated into the research work. All the published works are meeting the Open Access Publishing requirements and can be freely accessed, shared, modified, distributed and used in educational, commercial and non-commercial purposes under a Creative Commons Attribution 4.0 International License (CC BY 4.0). 